\title{
A concise synthesis of 3-(1-alkenyl)isoindolin-1-ones and 5-(1-alkenyl)pyrrol-2-ones by the intermolecular coupling reactions of $\mathrm{N}$-acyliminium ions with unactivated olefins
}

\author{
Nianhong Lu, Lihong Wang, Zhanshan Li and Wei Zhang ${ }^{*}$
}

\section{Full Research Paper}

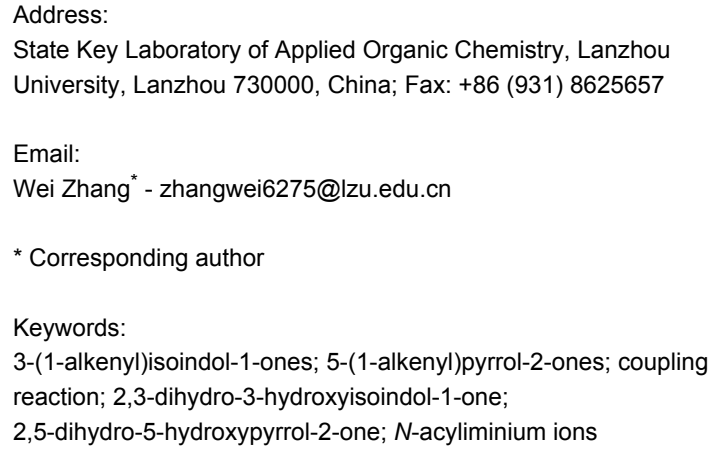
\author{
doi:10.3762/bjoc.8.21 \\ Received: 22 November 2011 \\ Accepted: 09 January 2012 \\ Published: 06 February 2012 \\ Associate Editor: M. P. Sibi
}

Beilstein J. Org. Chem. 2012, 8, 192-200.

(C) 2012 Lu et al; licensee Beilstein-Institut. License and terms: see end of document.

\begin{abstract}
A concise synthesis of 3-(1-alkenyl)isoindolin-1-ones and 5-(1-alkenyl)pyrrol-2-ones has been achieved by the coupling reactions of $\mathrm{N}$-acyliminium ions produced from 3-hydroxyisoindol-1-ones or 5-hydroxy-1-pyrrol-2-ones with unactivated olefins in the presence of $\mathrm{BF}_{3} \cdot \mathrm{OEt}_{2}$ at room temperature. For most of the olefins, the reactions afforded the $\mathrm{C}_{\mathrm{sp} 3}-\mathrm{C}_{\mathrm{sp} 2}$ cross-coupling products, but for the $\alpha$-methylstyrene and 1-hexene, the $\mathrm{C}_{\mathrm{sp} 3}-\mathrm{C}_{\mathrm{sp} 3}$ cross-coupling products were obtained.
\end{abstract}

\section{Introduction}

The coupling of alcohols with alkynes, aromatics and active methylene compounds has attracted great attention in recent years as an effective and environmentally benign strategy for the construction of carbon-carbon bonds with the concomitant loss of water. For example, the metal-catalyzed coupling of allyl, benzyl, and propargyl alcohols with terminal alkynes to give the doubly alkyl-substituted acetylenes [1-3]; the Brønsted acid and Lewis acid catalyzed coupling of alcohols with indoles to give the 3-alkyl-substituted indoles [4-6]; and the Brønsted acid and Lewis acid-catalyzed coupling of alcohols with 1,3-dicarbonyls to give the 2-alkyl-substituted 1,3-dicarbonyls [7-9]. All these reactions generally proceed by the addition of carbon cations to multiple bonds and subsequent deprotonation. In comparison, the reports for the coupling of alcohols with unactivated olefins to give the corresponding alkyl-substituted alkenes are rare. Lee recently reported a coupling of alcohols with olefins catalyzed by a ruthenium complex to give alkylsubstituted alkenes through the formation of $\mathrm{C}_{\mathrm{sp} 3}-\mathrm{C}_{\mathrm{sp} 2}$ bonds [10]; Liu reported the $\mathrm{FeCl}_{3} / \mathrm{TsOH}$ catalyzed coupling of diarylmethanol with styrenes to afford the alkyl-substituted styrenes 
[11]. We have long been interested in the reactions of $N$-acyliminium ions produced easily by the Brønsted acid and Lewis acid catalyzed dehydroxylation of $\alpha$-hydroxyamides [12-14]. The high electrophilicity of these species is very suitable for electrophilic addition to carbon-carbon multiple bonds. The coupling reactions of $\mathrm{N}$-acyliminium ions with various carbon nucleophiles, such as allylsilanes, alkylmetals, TMSCN, 1,3-dicarbonyls, isonitriles, enol derivatives and aromatics has been studied extensively [15-17]. Few reports are found to deal with the intermolecular coupling reactions of $\mathrm{N}$-acyliminium ions with unactivated olefins, although the intramolecular addition of acyliminium ions to olefins has been reported [18] The reported olefins that coupled with $N$-acyliminium ions were generally activated alkenes, such as 1-alkenylsilanes [19], 1-alkenylcoppers [20,21], 1-alkenylalanes [22] and 1-alkenylboronic acid, or esters [23,24] besides allylsilane. For example, Angst reported the coupling of styrylsilanes with $\mathrm{N}$-acyl-2chloroglycine esters catalyzed by $\mathrm{SnCl}_{4}$ to give the 3-styryl glycine derivatives in 1987 [19]; Wistrand reported the coupling of methyl 1-acyl-5-methoxy- $L$-proline with 1-alkenylcoppers catalyzed by $\mathrm{BF}_{3} \cdot \mathrm{OEt}_{2}$ to give methyl 1-acyl-5-(1-alkenyl)- $L$ proline in 1992 [20]; Menicagli reported the coupling of $\mathrm{N}$-acylisoquinolium chloride with di-isobutyl 1-hexenylalanes to give 1,2-dihydro-2-acyl-1-hexenylisoquinolines in 2008 [22]; Schaus reported the coupling of 1-alkenylboronates with 2-ethoxy- $N$-acylquinolines catalyzed by tartaric acid to produce 2-(1-alkenyl)- $N$-acylquinolines in 2011 [23]. We report here a concise synthesis of 3-(1-alkenyl)isoindolin-1-ones and 5-(1-alkenyl)pyrrol-2-ones by the cross-coupling reactions of $\mathrm{N}$-acyliminium ions derived from 3-hydroxyisoindol-1-ones or 5-hydroxypyrrol-2-ones with unactivated olefins such as styrene (2a) (Scheme 1 and Scheme 2).
Isoindolinones and pyrrolones are the core structures of numerous natural alkaloids [25-27] as well as many drug candidates [28-30]. Isoindolinones demonstrate a remarkably wide range of biological activities, including anti-inflammatory, antihypertensive, antipsychotic and antileukemic and antiviral effects [31-33]. Thus, many methods have been developed to synthesize 2- or 3-functionalized isoindolinones. Among them, only two reports dealt with the synthesis of 3-(1-alkenyl)isoindolin-1-one derivatives. One was, as mentioned above, by the $\mathrm{Cp}_{2} \mathrm{ZrCl}_{2}$ catalyzed coupling of $\mathrm{N}$-acyliminium ions with in situ generated dimethyl 1-alkenylalanes [24], another was performed by the palladium-catalyzed coupling of 2-iodobenzoyl chloride with aldimines and subsequent cyclization [34]. The results of our investigation have furnished another route to the synthesis of 3-(1-alkenyl)isoindolin-1-ones and 5-(1alkenyl)pyrrol-2-ones.

\section{Results and Discussion}

Two kinds of $N$-acyliminium ion precursors, 3-hydroxyisoindol-1-ones $(\mathbf{1} \mathbf{a}-\mathbf{c})$ and 5-hydroxypyrrol-2-ones $(\mathbf{5 a}, \mathbf{b})$ were easily prepared by the reduction of the parent phthalimide [35] and maleimide [36] derivatives. In order to explore the effects of the experimental conditions on the coupling reactions, the reaction of 1a with styrene (2a) was selected as a representative and carried out at room temperature under different conditions (Table 1). The use of a larger amount of catalyst led to an increase in the yield of the coupling product 3a (Table 1, entries 1-3). This observation is general for most of the intermolecular coupling reactions of $\mathrm{N}$-acyliminium ions with the weakest nucleophiles [14-16]. Of the catalysts examined, $\mathrm{BF}_{3} \cdot \mathrm{OEt}_{2}$ was very efficient for the formation of $\mathbf{3 a}$ compared to other catalysts such as $\mathrm{CF}_{3} \mathrm{SO}_{3} \mathrm{H}, \mathrm{CH}_{3} \mathrm{CO}_{2} \mathrm{H}, \mathrm{TiCl}_{4}, \mathrm{SnCl}_{4}$<smiles>O=C1c2ccccc2C(O)N1Cc1ccccc1</smiles>

$1 a$

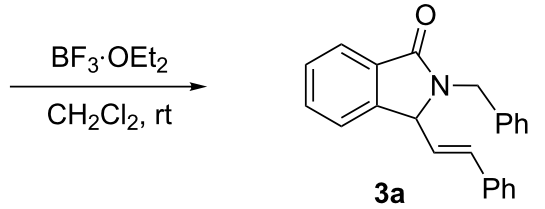

Scheme 1: Reaction of 3-hydroxyisoindol-1-one with styrene.
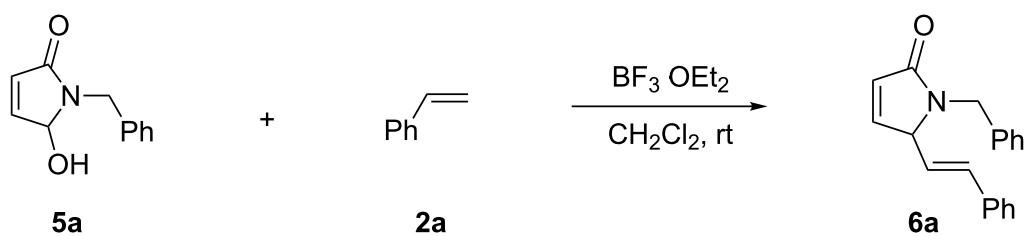

Scheme 2: Reaction of 5-hydroxypyrrol-1-one with styrene. 


\begin{tabular}{|c|c|c|c|c|c|}
\hline Entry & Solvent & Catalyst & $\begin{array}{l}t \\
(\mathrm{~h})\end{array}$ & $\begin{array}{l}T \\
\left({ }^{\circ} \mathrm{C}\right)\end{array}$ & $\begin{array}{l}\text { Yield }^{b} \\
(\%)\end{array}$ \\
\hline 1 & $\mathrm{CH}_{2} \mathrm{Cl}_{2}$ & 1.0 equiv $\mathrm{BF}_{3} \cdot \mathrm{OEt}_{2}$ & 1.0 & 25 & 65 \\
\hline 2 & $\mathrm{CH}_{2} \mathrm{Cl}_{2}$ & 1.5 equiv $\mathrm{BF}_{3} \cdot \mathrm{OEt}_{2}$ & 1.0 & 25 & 80 \\
\hline 3 & $\mathrm{CH}_{2} \mathrm{Cl}_{2}$ & 2.0 equiv $\mathrm{BF}_{3} \cdot \mathrm{OEt}_{2}$ & 1.0 & 25 & 83 \\
\hline 4 & $\mathrm{CH}_{2} \mathrm{Cl}_{2}$ & 2.0 equiv $\mathrm{CF}_{3} \mathrm{SO}_{3} \mathrm{H}$ & 1.0 & 25 & 50 \\
\hline 5 & $\mathrm{CH}_{2} \mathrm{Cl}_{2}$ & 2.0 equiv $\mathrm{CF}_{3} \mathrm{CO}_{2} \mathrm{H}$ & 1.0 & 25 & 37 \\
\hline 6 & $\mathrm{CH}_{2} \mathrm{Cl}_{2}$ & 2.0 equiv $\mathrm{TiCl}_{4}$ & 1.0 & 25 & 30 \\
\hline 7 & $\mathrm{CH}_{2} \mathrm{Cl}_{2}$ & 2.0 equiv $\mathrm{SnCl}_{4}$ & 1.0 & 25 & 25 \\
\hline 8 & $\mathrm{CH}_{2} \mathrm{Cl}_{2}$ & 2.0 equiv $\mathrm{InCl}_{3}$ & 1.0 & 25 & 21 \\
\hline 9 & $\mathrm{CH}_{3} \mathrm{CN}$ & 2.0 equiv $\mathrm{BF}_{3} \cdot \mathrm{OEt}_{2}$ & 1.0 & 25 & 66 \\
\hline 10 & $\mathrm{Et}_{2} \mathrm{O}$ & 2.0 equiv $\mathrm{BF}_{3} \cdot \mathrm{OEt}_{2}$ & 1.0 & 25 & 64 \\
\hline
\end{tabular}

${ }^{a}$ Reactions were carried out on $1.0 \mathrm{mmol}$ scale in $15.0 \mathrm{~mL}$ of solvent for $1.0 \mathrm{~h}$ with $1 \mathrm{a}(0.1 \mathrm{mmol}), \mathbf{2 a}(2.0 \mathrm{mmol})$ and catalyst $(2.0 \mathrm{mmol})$;

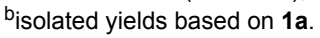

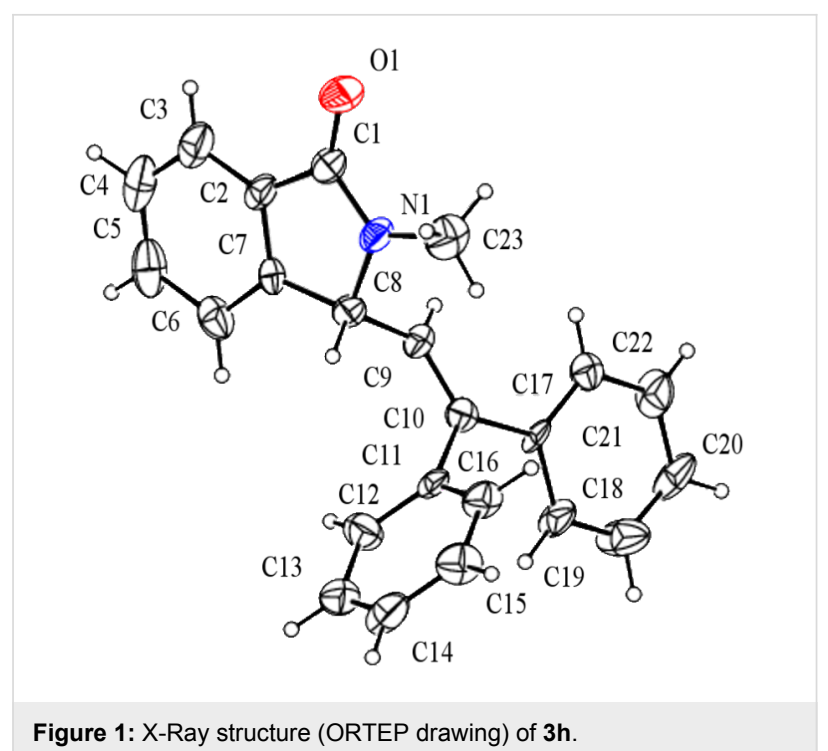

tion efficiency and selectivity appeared to be strongly dependent upon variation of the structure of the alkene component. The yields of the coupling adducts are seen to gradually decrease as the nucleophilicity of the alkene diminishes, as is exemplified by the yields recorded for the reactions between 1a and $\mathbf{1 b}$ and diphenyl ethylene, $\alpha$-methylstyrene and styrene (case of 1a: Table 2, entries 1-3 and case of 1b: Table 3, entries $1-3)$. The same trend is also observed in the less favorable case of 1c (Table 3, entries 8-10). Consistent with this reactivity profile, hexene gave only a moderate yield of adduct $4 \mathbf{b}$ when reacted with 1a (Table 2, entry 8 ). Likewise, alkenes bearing allylic protons prone to $\beta$-elimination, such as $\alpha$-methylstyrene and hexane, did not afford the "normal" $\mathrm{C}_{\mathrm{sp} 3}-\mathrm{C}_{\mathrm{sp} 2}$ vinylic adducts of type 3 , but instead the $\mathrm{C}_{\mathrm{sp} 3}-\mathrm{C}_{\mathrm{sp} 3}$ coupling products 4 were isolated (Table 2, entries 2 and 8 and Table 3, entries 2 and 9) much like the ene-type adducts of oxonium ion with olefins [37,38]. This means that these alkenes may be envisioned as surrogates of their corresponding, more expensive and less atom-economical, allylsilane derivatives, which are typically used in $\mathrm{N}$-acyliminium ion chemistry to produce amide compounds substituted with an $\alpha$-allyl group. The reactions of cyclic alkenes $(\mathbf{2 d}-\mathbf{g})$ with $\mathbf{1 a}, \mathbf{b}$ all gave the normal $\mathrm{C}_{\mathrm{sp} 3}-\mathrm{C}_{\mathrm{sp} 2}$ coupling products in moderate yields.<smiles>[R]C=C([R1])CC1c2ccccc2C(=O)N1[R1]</smiles> 
Table 2: The reactions of 3-hydroxyisoindol-1-one $1 \mathrm{a}$ with olefins 2 in the presence of $\mathrm{BF}_{3} \cdot \mathrm{OEt}_{2} \cdot{ }^{\mathrm{a}}$

Entry

Reactants

$\mathrm{R}^{2}$

$\mathrm{R}^{3}$

$\mathrm{R}^{4}$

$t(\mathrm{~h}) \quad T\left({ }^{\circ} \mathrm{C}\right)$

Product

Yield $^{\mathrm{b}}(\%)$
$1 \quad$ 1a $\quad \mathrm{PhCH}_{2}$

1

2b $\quad \mathrm{CH}_{3} \quad \mathrm{Ph} \quad \mathrm{H}$

$0.5 \quad 25$

2c $\mathrm{Ph} \quad \mathrm{Ph} \quad \mathrm{H}$

$0.25 \quad 25$<smiles>O=C1c2ccccc2C(/C=C/c2ccccc2)N1Cc1ccccc1</smiles>

3a<smiles>C=C(CC1c2ccccc2C(=O)N1Cc1ccccc1)c1ccccc1</smiles>

90

$\mathrm{H}$

4a<smiles>O=C1c2ccccc2C(C=C(c2ccccc2)c2ccccc2)N1Cc1ccccc1</smiles>

93<smiles>O=C1c2ccccc2C(C2=Cc3ccccc3C2)N1Cc1ccccc1</smiles>

3c

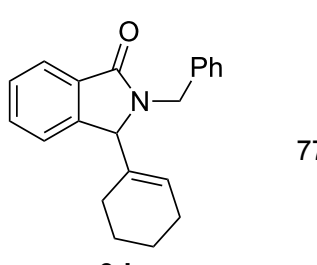

59

$\begin{array}{llllllll}6 & \mathbf{1 a} & \mathrm{PhCH}_{2} & \mathbf{2 f} & \mathrm{H} & -\left(\mathrm{CH}_{2}\right)_{3} \mathrm{O}- & 1.0 & 25\end{array}$

$\begin{array}{lll}7 & \text { 1a } & \mathrm{PhCH}_{2}\end{array}$

2g $\mathrm{H} \quad-\left(\mathrm{CH}_{2}\right)_{2} \mathrm{O}-$

$1.0 \quad 25$<smiles>O=C1c2ccccc2C(C2=COCCC2)N1Cc1ccccc1</smiles>

$3 e$<smiles>O=C1c2ccccc2C(C2=COCC2)N1Cc1ccccc1</smiles> 
Table 2: The reactions of 3-hydroxyisoindol-1-one $1 \mathrm{a}$ with olefins 2 in the presence of $\mathrm{BF}_{3} \cdot \mathrm{OEt}_{2}{ }^{\mathrm{a}}$ (continued)

$\begin{array}{llllllll}\text { 1a } & \mathrm{PhCH}_{2} & \text { 2h } & \mathrm{H} & \mathrm{H} & n-\mathrm{Bu} & 2.0 & 25\end{array}$

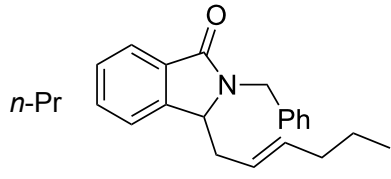

$4 \mathrm{~b}$

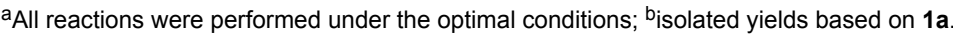

Table 3: The reactions of 3-hydroxyisoindol-1-one $(\mathbf{1 b}, \mathbf{c})$ with olefins 2 in the presence of $\mathrm{BF}_{3} \cdot \mathrm{OEt}_{2}{ }^{\mathrm{a}}$

Entry

Reactants

$\mathrm{R}^{1}$

$R^{2} \quad R^{3} \quad R^{4}$

11 1b $\quad \mathrm{CH}_{3}$

2a $\mathrm{H} \quad \mathrm{Ph} \quad \mathrm{H}$

$t(\mathrm{~h}) \quad T\left({ }^{\circ} \mathrm{C}\right)$

Yield $^{\mathrm{b}}(\%)$

$\mathrm{R}^{5}$

$0.5 \quad 25$

2b $\begin{array}{llll}\mathrm{CH}_{3} & \mathrm{Ph} & \mathrm{H}\end{array}$

$0.5 \quad 25$<smiles>CN1C(=O)c2ccccc2C1/C=C/c1ccccc1</smiles>

70

3g

21 1b $\quad \mathrm{CH}_{3}$

$\mathrm{H}$<smiles>C=C(CC1c2ccccc2C(=O)N1C)c1ccccc1</smiles>

4c<smiles>CN1C(=O)c2ccccc2C1C=C(c1ccccc1)c1ccccc1</smiles>

94

$3 h$<smiles>CN1C(=O)c2ccccc2C1C1=Cc2ccccc2C1</smiles>

$3 \mathbf{i}$

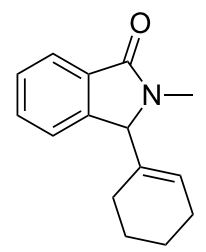


Table 3: The reactions of 3-hydroxyisoindol-1-one $(\mathbf{1 b}, \mathbf{c})$ with olefins 2 in the presence of $\mathrm{BF}_{3} \cdot \mathrm{OEt}_{2} \cdot{ }^{\mathrm{a}}$ (continued)

1b $\mathrm{CH}_{3}$

$7 \quad 1 b \quad \mathrm{CH}_{3}$

1c $\mathrm{H}$

$9 \quad 1 \mathrm{c} \quad \mathrm{H}$

10 1c $\mathrm{H}$

11 1c $\mathrm{H}$ 2f

$-\left(\mathrm{CH}_{2}\right)_{3} \mathrm{O}-$

$2 \mathrm{~g}$

$-\left(\mathrm{CH}_{2}\right)_{2} \mathrm{O}-$

1.0

25

2a $\mathrm{H} \quad \mathrm{Ph} \quad \mathrm{H}$

$1.0 \quad 25$

2b $\quad \mathrm{CH}_{3} \quad \mathrm{Ph} \quad \mathrm{H}$

$1.0 \quad 25$

2c $\mathrm{Ph}$

$\mathrm{Ph}$

$\mathrm{H}$

$1.0 \quad 25$

2d $\mathrm{H} \quad-\mathrm{CH}_{2} \mathrm{C}_{6} \mathrm{H}_{4}-$<smiles>CN1C(=O)c2ccccc2C1C1=COCCC1</smiles>

48

3k<smiles>O=C1c2ccccc2C(C2=COCC2)N1Cc1ccccc1</smiles>

3I<smiles>O=C1NC(/C=C/c2ccccc2)c2ccccc21</smiles>

$3 \mathrm{~m}$<smiles>C=C(CC1NC(=O)c2ccccc21)c1ccccc1</smiles>

4d<smiles>O=C1NC(C=C(c2ccccc2)c2ccccc2)c2ccccc21</smiles>

73

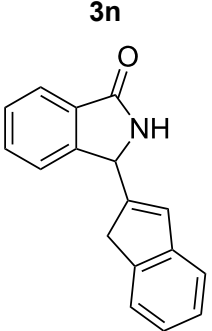

30

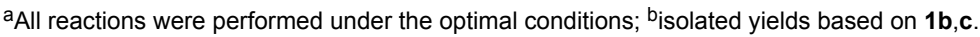

The coupling reactions were examined under the same conditions with alternate substrates $(\mathbf{5 a}, \mathbf{b})$, and olefins $(\mathbf{2} \mathbf{a}-\mathbf{c})$ (Scheme 4). All these reactions gave the cross-coupling products (Table 4). As compared with 1a-c, the rates of the coupling reactions of $\mathbf{5 a}, \mathbf{b}$ with $\mathbf{2 a}-\mathbf{c}$ were somewhat slower and the yields of the corresponding products were also decreased, prob- ably as a result of both the limited nucleophilicity parameter of the alkenes [39] and the lower stability of the transient $\mathrm{N}$-acyliminium intermediate derived from $\mathbf{5 a}, \mathbf{b}$. Similarly to the reactions of $\mathbf{1 a}-\mathbf{c}$ with $\alpha$-methylstyrene (2b), the reactions of $\mathbf{5 a}, \mathbf{b}$ with $\mathbf{2 b}$ also gave the $\mathrm{C}_{\mathrm{sp} 3}-\mathrm{C}_{\mathrm{sp} 3}$ coupling products $\mathbf{7 a}, \mathbf{b}$ instead of the $\mathrm{C}_{\mathrm{sp} 3}-\mathrm{C}_{\mathrm{sp} 2}$ coupling products. 


\begin{tabular}{|c|c|c|c|c|c|c|c|c|c|}
\hline \multirow[t]{2}{*}{ Entry } & \multicolumn{5}{|c|}{ Reactants } & \multirow[t]{2}{*}{$t(\mathrm{~h})$} & \multirow[t]{2}{*}{$T\left({ }^{\circ} \mathrm{C}\right)$} & \multirow[t]{2}{*}{ Product } & \multirow[t]{2}{*}{ Yield $^{\mathrm{b}}(\%)$} \\
\hline & & $\mathrm{R}^{1}$ & & $\mathrm{R}^{2}$ & $\mathrm{R}^{3}$ & & & & \\
\hline 1 & $5 a$ & $\mathrm{PhCH}_{2}$ & $2 a$ & $\mathrm{H}$ & $\mathrm{Ph}$ & 2.0 & 25 & $6 a$ & 55 \\
\hline 2 & $5 a$ & $\mathrm{PhCH}_{2}$ & $2 b$ & $\mathrm{CH}_{3}$ & $\mathrm{Ph}$ & 2.0 & 25 & $7 a$ & 82 \\
\hline 3 & $5 a$ & $\mathrm{PhCH}_{2}$ & $2 c$ & $\mathrm{Ph}$ & $\mathrm{Ph}$ & 2.0 & 25 & $6 b$ & 72 \\
\hline 4 & $5 b$ & $\mathrm{CH}_{3}$ & $2 a$ & $\mathrm{CH}_{3}$ & $\mathrm{Ph}$ & 2.0 & 25 & $7 b$ & 72 \\
\hline 5 & $5 b$ & $\mathrm{CH}_{3}$ & $2 b$ & $\mathrm{Ph}$ & $\mathrm{Ph}$ & 2.0 & 25 & $6 c$ & 65 \\
\hline
\end{tabular}

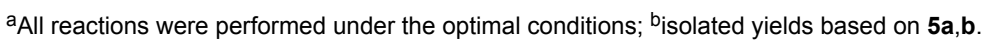

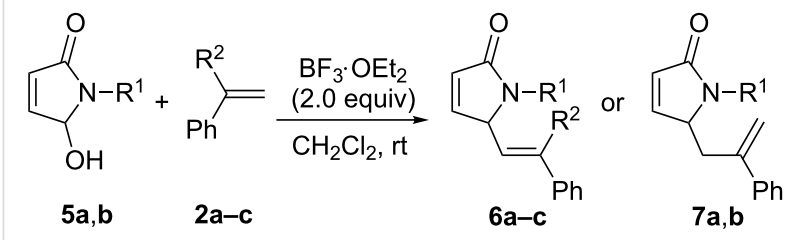

Scheme 4: Reactions of 5-hydroxypyrrol-1-ones with olefins in the presence of $\mathrm{BF}_{3} \cdot \mathrm{OEt}_{2}$.

\section{Conclusion}

In summary, we have developed a concise route for the synthesis of 3-(1-alkenyl)isoindolin-1-ones and 5-(1alkenyl)pyrrol-2-ones by the coupling reactions of $\mathrm{N}$-acyliminium ions derived from 3-hydroxyisoindol-1-ones or 5 -hydroxypyrrol-2-ones with unactivated olefins in the presence of $\mathrm{BF}_{3} \cdot \mathrm{OEt}_{2}$ at room temperature. For most of the olefins, the reactions afforded the $\mathrm{C}_{\mathrm{sp} 3}-\mathrm{C}_{\mathrm{sp} 2}$ cross-coupling products, but for $\alpha$-methylstyrene and 1 -hexene, the $\mathrm{C}_{\mathrm{sp} 3}-\mathrm{C}_{\mathrm{sp} 3}$ crosscoupling products were produced.

\section{Experimental}

\section{General information}

All reagents were purchased from commercial suppliers and used without further purification. All solvents were dried and redistilled before use. Flash chromatography was carried out with silica gel (200-300 mesh). Analytical TLC was performed with silica gel $\mathrm{GF}_{254}$ plates, and the products were visualized by UV detection. Melting points were determined on a Yanagimoto melting point apparatus and are uncorrected. ${ }^{1} \mathrm{H}$ and ${ }^{13} \mathrm{C}$ NMR spectra were recorded on a Bruker AM-400 NMR or a Bruker DRX-300 NMR spectrometer in $\mathrm{CDCl}_{3}$ with TMS as an internal standard. EIMS were recorded with a HP 5988 A mass spectrometer. HRMS (ESI) were measured on a Bruker Dattonics APEX 47e mass spectrometer.

\section{General procedure for the coupling reactions}

To a solution of $1 \mathbf{a}(1.0 \mathrm{mmol})$ and olefin $\mathbf{2 a}(2.0 \mathrm{mmol})$ in $15 \mathrm{~mL}$ of anhydrous methylene dichloride, $\mathrm{BF}_{3} \cdot \mathrm{OEt}_{2}$ (2.0 mmol) was added at $25^{\circ} \mathrm{C}$ in one portion under stirring. After continued stirring at $25^{\circ} \mathrm{C}$ until 1 a disappeared (monitored by TLC), the reaction was quenched with water. The mixture was separated and the aqueous phase was extracted with methylene dichloride $(10 \mathrm{~mL})$. The combined organic layers were washed with water $(20 \mathrm{~mL})$, dried with anhydrous $\mathrm{Na}_{2} \mathrm{SO}_{4}$ and concentrated in vacuo. The residue was separated by silicagel column chromatography, eluted by hexane/acetone $(10: 1 \mathrm{v} / \mathrm{v})$, to give the corresponding product $\mathbf{3 a}$.

(E)-2-Benzyl-3-(2-phenylethenyl)isoindolin-1-one (3a): Colorless syrup; ${ }^{1} \mathrm{H}$ NMR $\left(400 \mathrm{MHz}, \mathrm{CDCl}_{3}\right) \delta 4.22(\mathrm{~d}, J=$ $14.8 \mathrm{~Hz}, 1 \mathrm{H}), 4.90(\mathrm{~d}, J=9.2 \mathrm{~Hz}, 1 \mathrm{H}), 5.33(\mathrm{~d}, J=14.8 \mathrm{~Hz}$, $1 \mathrm{H}), 5.82(\mathrm{dd}, J=9.2 \mathrm{~Hz}, 15.6 \mathrm{~Hz}, 1 \mathrm{H}), 6.77(\mathrm{~d}, J=15.6 \mathrm{~Hz}$, $1 \mathrm{H}), 7.28-7.37(\mathrm{~m}, 11 \mathrm{H}), 7.50-7.55(\mathrm{~m}, 2 \mathrm{H}), 7.92(\mathrm{dd}, J=$ $1.6 \mathrm{~Hz}, 6.4 \mathrm{~Hz}, 1 \mathrm{H}) \mathrm{ppm} ;{ }^{13} \mathrm{C} \mathrm{NMR}\left(100 \mathrm{MHz}, \mathrm{CDCl}_{3}\right) \delta 44.1$, $62.7,123.2,123.8,125.6,126.7$ (2C), 127.5, $128.4(2 \mathrm{C}), 128.5$ (2C), 128.6 (2C), 128.7, 128.7, 131.7, 131.8, 135.7, 135.9, $137.4,144.5,168.0(\mathrm{CO}) \mathrm{ppm}$; EIMS $\mathrm{m} / \mathrm{z}$ (\% relative intensity): 325 (56), 310 (29), 234 (89), 220 (31), 149 (46), 91 (45), 57 (53), 44 (100); HRMS-ESI $(\mathrm{m} / z):[\mathrm{M}+\mathrm{H}]^{+}$calculated for $\mathrm{C}_{23} \mathrm{H}_{20} \mathrm{NO}$, 326.1540; found, 326.1536 .

2-Benzyl-3-(2-phenyl-2-propenyl)isoindolin-1-one (4a): Colorless solid, mp $69-72{ }^{\circ} \mathrm{C} ;{ }^{1} \mathrm{H}$ NMR $\left(400 \mathrm{MHz}, \mathrm{CDCl}_{3}\right)$ $\delta 2.54(\mathrm{dd}, J=9.2 \mathrm{~Hz}, 14.0 \mathrm{~Hz}, 1 \mathrm{H}), 3.40(\mathrm{dd}, J=4.0 \mathrm{~Hz}$, $14.0 \mathrm{~Hz}, 1 \mathrm{H}), 4.24(\mathrm{~d}, J=15.6 \mathrm{~Hz}, 1 \mathrm{H}), 4.39$ (dd, $J=4.0 \mathrm{~Hz}$, $9.2 \mathrm{~Hz}, 1 \mathrm{H}), 5.00(\mathrm{~s}, 1 \mathrm{H}), 5.38(\mathrm{~s}, 1 \mathrm{H}), 5.40(\mathrm{~d}, J=15.6 \mathrm{~Hz}$, $1 \mathrm{H}), 7.20-7.31(\mathrm{~m}, 11 \mathrm{H}), 7.41(\mathrm{t}, J=4.0 \mathrm{~Hz}, 2 \mathrm{H}), 7.86(\mathrm{t}, J=$ $4.0 \mathrm{~Hz}, 1 \mathrm{H}) \mathrm{ppm} ;{ }^{13} \mathrm{C} \mathrm{NMR}\left(100 \mathrm{MHz}, \mathrm{CDCl}_{3}\right) \delta 38.1,44.1$, 56.9, 116.9, 123.2, 123.7, 126.1 (2C), 127.6, 127.8, 128.1, 128.1 (2C), 128.5 (2C), 128.8 (2C), 130.9, 131.8, 137.0, 139.8, 143.6, 145.2, $168.4(\mathrm{CO}) \mathrm{ppm}$; MS $\mathrm{m} / z$ (\% relative intensity): 339 (1), 253 (4), 237 (6), 222 (100), 197 (5), 149 (13), 91 (71); 
HRMS-ESI $(m / z):[\mathrm{M}+\mathrm{H}]^{+}$calcd for $\mathrm{C}_{24} \mathrm{H}_{22} \mathrm{NO}, 340.1696$; found, 340.1699 .

2-Benzyl-3-cyclohexenylisoindolin-1-one (3d): Colorless solid, mp 109-112 ${ }^{\circ} \mathrm{C} ;{ }^{1} \mathrm{H}$ NMR (400 $\left.\mathrm{MHz}, \mathrm{CDCl}_{3}\right)$ $\delta 1.15-1.19(\mathrm{~m}, 1 \mathrm{H}), 1.38-1.43(\mathrm{~m}, 3 \mathrm{H}), 1.50-1.59(\mathrm{~m}, 2 \mathrm{H})$, $2.13(\mathrm{t}, J=2.4 \mathrm{~Hz}, 2 \mathrm{H}), 4.06(\mathrm{~d}, J=14.8 \mathrm{~Hz}, 1 \mathrm{H}), 4.71(\mathrm{~s}, 1 \mathrm{H})$, $5.19(\mathrm{~d}, J=14.8 \mathrm{~Hz}, 1 \mathrm{H}), 5.93(\mathrm{~s}, 1 \mathrm{H}), 7.26-7.30(\mathrm{~m}, 5 \mathrm{H})$, $7.41-7.50(\mathrm{~m}, 3 \mathrm{H}), 7.87(\mathrm{~d}, J=7.2 \mathrm{~Hz}, 1 \mathrm{H}) \mathrm{ppm} ;{ }^{13} \mathrm{C} \mathrm{NMR}$ $\left(100 \mathrm{MHz}, \mathrm{CDCl}_{3}\right) \delta 21.8,22.0,22.2,25.4,43.9,66.7,122.4$, 123.4, 127.3, 128.1, 128.4 (4C), 130.2, 131.4, 132.3, 133.4, 137.4, 144.4, $168.3(\mathrm{CO}) \mathrm{ppm}$; MS $m / z$ (\% relative intensity): 303 (64), 222 (27), 199 (70), 183 (6), 170 (12), 157 (15), 129 (27), 91 (100), 40 (37); HRMS-ESI $(\mathrm{m} / z):[\mathrm{M}+\mathrm{H}]^{+}$calcd for $\mathrm{C}_{21} \mathrm{H}_{22} \mathrm{NO}, 304.1696$; found, 304.1691.

(E)-2-Benzyl-3-(hex-2-enyl)isoindolin-1-one (4b): Colorless syrup; ${ }^{1} \mathrm{H}$ NMR $\left(400 \mathrm{MHz}, \mathrm{CDCl}_{3}\right) \delta 0.74(\mathrm{t}, J=7.2 \mathrm{~Hz}, 3 \mathrm{H})$, $1.25-1.87(\mathrm{~m}, 2 \mathrm{H}), 1.79-1.86(\mathrm{~m}, 2 \mathrm{H}), 2.55-2.70(\mathrm{~m}, 2 \mathrm{H}), 4.17$ $(\mathrm{d}, J=15.2 \mathrm{~Hz}, 1 \mathrm{H}), 4.39(\mathrm{dd}, J=4.0 \mathrm{~Hz}, 5.6 \mathrm{~Hz}, 1 \mathrm{H})$, $4.91-4.98(\mathrm{~m}, 1 \mathrm{H}), 5.36-5.42(\mathrm{~m}, 1 \mathrm{H}), 5.42(\mathrm{~d}, J=15.2 \mathrm{~Hz}$, 1H), 7.28-7.32 (m, 5H), 7.37 (d, $J=7.2 \mathrm{~Hz}, 1 \mathrm{H}), 7.43-7.53(\mathrm{~m}$, 2H), $7.88(\mathrm{~d}, J=7.6 \mathrm{~Hz}, 1 \mathrm{H}) \mathrm{ppm} ;{ }^{13} \mathrm{C} \mathrm{NMR}(100 \mathrm{MHz}$, $\left.\mathrm{CDCl}_{3}\right) \delta 13.4,22.3,34.1,34.5,43.9,58.4,122.4(2 \mathrm{C}), 123.7$, 127.5, 128.0, 128.1 (2C), 128.7 (2C), 131.2, 132.4, 135.4, $137.2,145.1,168.5(\mathrm{CO}) \mathrm{ppm}$; MS $m / z$ (\% relative intensity): 305 (4), 223 (18), 222 (100), 186 (6), 172 (6), 132 (8), 104 (5), 91 (89); HRMS-ESI $(\mathrm{m} / z):[\mathrm{M}+\mathrm{H}]^{+}$calcd for $\mathrm{C}_{21} \mathrm{H}_{24} \mathrm{NO}$, 306.1853; found, 306.1851 .

3-(2,2-Diphenylethenyl)-2-methylisoindolin-1-one (3h): Colorless solid, mp $146-148{ }^{\circ} \mathrm{C} ;{ }^{1} \mathrm{H}$ NMR $\left(400 \mathrm{MHz}, \mathrm{CDCl}_{3}\right)$ $\delta 3.08(\mathrm{~s}, 3 \mathrm{H}), 5.01(\mathrm{~d}, J=10.0 \mathrm{~Hz}, 1 \mathrm{H}), 5.71(\mathrm{~d}, J=10.0 \mathrm{~Hz}$, $1 \mathrm{H}), 7.25-7.27(\mathrm{~m}, 5 \mathrm{H}), 7.38-7.46(\mathrm{~m}, 5 \mathrm{H}), 7.47-7.52(\mathrm{~m}, 3 \mathrm{H})$, $7.83(\mathrm{~d}, J=7.6 \mathrm{~Hz}, 1 \mathrm{H}) \mathrm{ppm} ;{ }^{13} \mathrm{C} \mathrm{NMR}\left(100 \mathrm{MHz}, \mathrm{CDCl}_{3}\right)$ $\delta 27.5,61.3,122.8,123.4,124.3,127.2$ (2C), 127.9, 128.1, 128.3 (3C), 128.8 (2C), 129.5 (2C), 131.3, 132.3, 138.5, 140.4, $144.5,148.1,168.0(\mathrm{CO}) \mathrm{ppm}$; MS $\mathrm{m} / z$ (\% relative intensity): 325 (28), 310 (15), 294 (9), 265 (5), 248 (11), 220 (18), 188 (10), 178 (11), 165 (13), 149 (37), 91 (30), 57 (63), 43 (100); HRMS-ESI $(\mathrm{m} / \mathrm{z}):[\mathrm{M}+\mathrm{H}]^{+}$calcd for $\mathrm{C}_{23} \mathrm{H}_{20} \mathrm{NO}, 326.1540$; found, 326.1545 .

Crystal data for $3 \mathrm{~h}$ (recrystallized from ethanol): $\mathrm{C}_{23} \mathrm{H}_{19} \mathrm{NO}, M_{\mathrm{r}}=325.39$. Monoclinic, $a=17.373(11) \AA, b=$ 17.241(11) $\AA, c=24.421(16) \AA, \beta=91.219(9), V=7313(8) \AA^{3}$, colorless plates, $\rho=1.182 \mathrm{~g} \mathrm{~cm}^{-3}, T=296(2) \mathrm{K}$, space group $P 2(1) / c, Z=4, \mu($ Mo K $\alpha)=0.084 \mathrm{~mm}^{-1}, 2 \theta_{\max }=51^{\circ}, 9126$ reflections measured, 3995 unique $\left(R_{\mathrm{int}}=0.0696\right)$, which were used in all calculations. The final $\mathrm{w} R\left(F^{2}\right)$ was 0.1427 (for all data), $R_{1}=0.0764$. CCDC file No. 835330 .
3-(3,4-Dihydro-2H-pyran-5-yl)-2-methylisoindolin-1-one (3k): Colorless solid, mp 94-97 ${ }^{\circ} \mathrm{C} ;{ }^{1} \mathrm{H}$ NMR $(400 \mathrm{MHz}$, $\left.\mathrm{CDCl}_{3}\right) \delta 1.20-1.27(\mathrm{~m}, 1 \mathrm{H}), 1.40-1.47(\mathrm{~m}, 1 \mathrm{H}), 1.71-1.79(\mathrm{~m}$, $2 \mathrm{H}), 3.00(\mathrm{~s}, 3 \mathrm{H}), 3.92-4.04(\mathrm{~m}, 2 \mathrm{H}), 4.65(\mathrm{~s}, 1 \mathrm{H}), 6.79(\mathrm{~s}, 1 \mathrm{H})$, $7.36(\mathrm{~d}, J=7.2 \mathrm{~Hz}, 1 \mathrm{H}), 7.44(\mathrm{t}, J=7.2 \mathrm{~Hz}, 1 \mathrm{H}), 7.53$ (t, $J=$ $7.2 \mathrm{~Hz}, 1 \mathrm{H}), 7.81(\mathrm{~d}, J=7.2 \mathrm{~Hz}, 1 \mathrm{H}) \mathrm{ppm} ;{ }^{13} \mathrm{C}$ NMR $(100$ $\left.\mathrm{MHz}, \mathrm{CDCl}_{3}\right) \delta 17.0,21.5,26.6,65.2,66.0,108.2,122.2$, $123.0,128.1,131.3,132.7,144.2,144.6,168.2$ (CO) ppm; MS $m / z$ (\% relative intensity): 229 (100), 200 (47), 186 (35), 172 (54), 146 (51), 128 (20), 115 (17), 91 (24); HRMS-ESI $(m / z):[\mathrm{M}+\mathrm{H}]^{+}$calcd for $\mathrm{C}_{14} \mathrm{H}_{16} \mathrm{NO}_{2}, 230.1176$; found, 230.1175 .

(E)-1-Benzyl-5-(2-phenylethenyl)-1H-pyrrol-2(5H)-one (6a): Colorless syrup; ${ }^{1} \mathrm{H}$ NMR $\left(400 \mathrm{MHz}, \mathrm{CDCl}_{3}\right) \delta 4.08$ (d, $J=$ $14.8 \mathrm{~Hz}, 1 \mathrm{H}), 4.54(\mathrm{~d}, J=9.2 \mathrm{~Hz}, 1 \mathrm{H}), 5.12(\mathrm{~d}, J=14.8 \mathrm{~Hz}$, $1 \mathrm{H}), 5.69(\mathrm{dd}, J=9.2 \mathrm{~Hz}, 15.6 \mathrm{~Hz}, 1 \mathrm{H}), 6.26(\mathrm{dd}, J=1.6 \mathrm{~Hz}$, $5.6 \mathrm{~Hz}, 1 \mathrm{H}), 6.59(\mathrm{~d}, J=15.6 \mathrm{~Hz}, 1 \mathrm{H}), 6.96(\mathrm{dd}, J=1.6 \mathrm{~Hz}$, $6.0 \mathrm{~Hz}, 1 \mathrm{H}), 7.23-7.35(\mathrm{~m}, 8 \mathrm{H}), 7.40(\mathrm{dd}, J=1.6 \mathrm{~Hz}, 8.0 \mathrm{~Hz}$, $2 \mathrm{H}) \mathrm{ppm} ;{ }^{13} \mathrm{C} \mathrm{NMR}\left(100 \mathrm{MHz}, \mathrm{CDCl}_{3}\right) \delta 42.4,64.8,126.1$, $126.6,127.4,128.0,128.2,128.6(2 \mathrm{C}), 128.7$ (2C), 128.7 (2C), 128.9 (2C), 135.7, 137.6, 146.6, 170.9 (CO) ppm; MS m/z (\% relative intensity): 275 (22), 190 (11), 189 (100), 184 (30), 161 (29), 160 (39), 132 (37), 119 (22), 104 (48), 91 (21); HRMS-ESI $(\mathrm{m} / \mathrm{z}):[\mathrm{M}+\mathrm{H}]^{+}$calcd for $\mathrm{C}_{19} \mathrm{H}_{18} \mathrm{NO}, 276.1383$; found, 276.1385 .

\section{Supporting Information}

\section{Supporting Information File 1}

Characterization data of the title compounds, ${ }^{1} \mathrm{H}$ NMR and

${ }^{13} \mathrm{C}$ NMR spectra.

[http://www.beilstein-journals.org/bjoc/content/ supplementary/1860-5397-8-21-S1.pdf]

\section{Supporting Information File 2}

$\mathrm{X}$-ray data for compound $\mathbf{3 h}$.

[http://www.beilstein-journals.org/bjoc/content/ supplementary/1860-5397-8-21-S2.cif]

\section{Acknowledgements}

This work was financially supported by the National Natural Science Foundation of China (20872056).

\section{References}

1. Ren, K.; Li, P.; Wang, L.; Zhang, X. Tetrahedron 2011, 67, 2753-2759. doi:10.1016/j.tet.2011.02.050

2. Wang, T.; Chen, X.-L.; Chen, L.; Zhan, Z.-P. Org. Lett. 2011, 13, 3324-3327. doi:10.1021/ol201054z 
3. Xiang, S.-K.; Zhang, L.-H.; Jiao, N. Chem. Commun. 2009, 6487-6489. doi:10.1039/B911905A

4. Wu, Y.-C.; Li, H.-J.; Liu, L.; Demoulin, N.; Liu, Z.; Wang, D.; Chen, Y.-J. Adv. Synth. Catal. 2011, 353, 907-912. doi:10.1002/adsc.201000930

5. Sanz, R.; Miguel, D.; Martínez, A.; Gohain, M.; García-García, P.; Fernández-Rodríguez, M. A.; Álvarez, E.; Rodríguez, F. Eur. J. Org. Chem. 2010, 7027-7039. doi:10.1002/ejoc.201001055

6. Davoust, M.; Kitching, J. A.; Fleming, M. J.; Lautens, M. Chem.-Eur. J. 2010, 16, 50-54. doi:10.1002/chem.200902694

7. Liu, P. N.; Dang, L.; Wang, Q. W.; Zhao, S. L.; Xia, F.; Ren, Y. J.; Gong, X. Q.; Chen, J. Q. J. Org. Chem. 2010, 75, 5017-5030. doi:10.1021/jo100517k

8. Reddy, C. R.; Vijaykumar, J.; Grée, R. Synthesis 2010, 3715-3723. doi:10.1055/s-0030-1258214

9. Theerthagiri, P.; Lalitha, A. Tetrahedron Lett. 2010, 51, 5454-5458. doi:10.1016/j.tetlet.2010.08.019

10. Lee, D.-H.; Kwon, K.-H.; Yi, C. S. Science 2011, 333, 1613-1616. doi:10.1126/science.1208839

11. Liu, Z.-Q.; Zhang, Y.; Zhao, L.; Li, Z.; Wang, J.; Li, H.; Wu, L.-M. Org. Lett. 2011, 13, 2208-2211. doi:10.1021/ol200372y

12. Zhang, W.; Zheng, A.; Liu, Z.; Yang, L.; Liu, Z. Tetrahedron Lett. 2005, 46. doi:10.1016/j.tetlet.2005.06.097

13. Zhang, W.; Huang, L.; Wang, J. Synthesis 2006, 2053-2063. doi:10.1055/s-2006-942372

14. Zhou, Y.; Qian, L.; Zhang, W. Synlett 2009, 843-847. doi:10.1055/s-0028-1087955

15. Yazici, A.; Pyne, S. G. Synthesis 2009, 339-368. doi:10.1055/s-0028-1083325

16. Yazici, A.; Pyne, S. G. Synthesis 2009, 513-541. doi:10.1055/s-0028-1083346

17. Speckamp, W. N.; Moolenaar, M. J. Tetrahedron 2000, 56, 3817-3856. doi:10.1016/S0040-4020(00)00159-9

18. Maryanoff, B. E.; Zhang, H.-C.; Cohen, J. H.; Turchi, I. J.; Maryanoff, C. A. Chem. Rev. 2004, 104, 1431-1628. doi:10.1021/cr0306182

19. Angst, C. Pure Appl. Chem. 1987, 59, 373-380. doi:10.1351/pac198759030373

20. Thaning, M.; Wistrand, L.-G. Acta Chem. Scand. 1992, 46, 194-199. doi:10.3891/acta.chem.scand.46-0194

21. McClure, K. F.; Renold, P.; Kemp, D. S. J. Org. Chem. 1995, 60, 454-457. doi:10.1021/jo00107a028

22. Signore, G.; Malanga, C.; Menicagli, R. Tetrahedron 2008, 64 , 197-203. doi:10.1016/j.tet.2007.10.077

23. Kodama, T.; Moquist, P. N.; Schaus, S. E. Org. Lett. 2011, 13, 6316-6319. doi:10.1021/ol2028702

24. Morgan, I. R.; Yazici, A.; Pyne, S. G. Tetrahedron 2008, 64, 1409-1419. doi:10.1016/j.tet.2007.11.046

25. Scherlach, K.; Schuemann, J.; Dahse, H.-M.; Hertweck, C. J. Antibiot. 2010, 63, 375-377. doi:10.1038/ja.2010.46

26. Chen, J.; Huang, P.-Q.; Queneau, Y. J. Org. Chem. 2009, 74, 7457-7463. doi:10.1021/j0901557h

27. Lamblin, M.; Couture, A.; Deniau, E.; Grandclaudon, P. Org. Biomol. Chem. 2007, 5, 1466-1471. doi:10.1039/B701661A

28. Sorbera, L. A.; Leeson, P. A.; Silvestre, J.; Castaner, J. Drugs Future 2001, 26, 651-657. doi:10.1358/dof.2001.026.07.630003

29. Wada, T.; Fukuda, N. Psychopharmacology (Berlin) 1991, 103, 314-322. doi:10.1007/BF02244284

30. Kawasuji, T.; Fuji, M.; Yoshinaga, T.; Sato, A.; Fujiwara, T.; Kiyama, R. Bioorg. Med. Chem. 2007, 15, 5487-5492.

doi:10.1016/j.bmc.2007.05.052
31. Zhuang, Z.-P.; Kung, M.-P.; Mu, M.; Kung, H. F. J. Med. Chem. 1998, 41, 157-166. doi:10.1021/jm970296s

32. Norman, M. H.; Minick, D. J.; Rigdon, G. C. J. Med. Chem. 1996, 39, 149-157. doi:10.1021/jm9502201

33. De Clercq, E. J. Med. Chem. 1995, 38, 2491-2517. doi:10.1021/jm00014a001

34. Cho, C. S.; Wu, X.; Jiang, L. H.; Shim, S. C.; Choi, H.-J.; Kim, T. J. J. Heterocycl. Chem. 1998, 35, 265-268. doi:10.1002/jhet.5570350147

35. Horii, Z.-I.; Iwata, C.; Tamura, Y. J. Org. Chem. 1961, 26, 2273-2276. doi:10.1021/jo01351a031

36. Mase, N.; Nishi, T.; Hiyoshi, M.; Ichihara, K.; Bessho, J.; Yoda, H.; Takabe, K. J. Chem. Soc., Perkin Trans. 1 2002, 707-709. doi:10.1039/B200729K

37. Mikami, K.; Kishino, H. J. Chem. Soc., Chem. Commun. 1993, 1843-1844. doi:10.1039/C39930001843

38. Mikami, K.; Shimizu, M. Chem. Rev. 1992, 92, 1021-1050. doi:10.1021/cr00013a014

39. Mayr, H.; Kempf, B.; Ofial, A. R. Acc. Chem. Res. 2003, 36, 66-77. doi:10.1021/ar020094c

\section{License and Terms}

This is an Open Access article under the terms of the Creative Commons Attribution License

(http://creativecommons.org/licenses/by/2.0), which permits unrestricted use, distribution, and reproduction in any medium, provided the original work is properly cited.

The license is subject to the Beilstein Journal of Organic Chemistry terms and conditions:

(http://www.beilstein-journals.org/bjoc)

The definitive version of this article is the electronic one which can be found at: doi:10.3762/bjoc. 8.21 